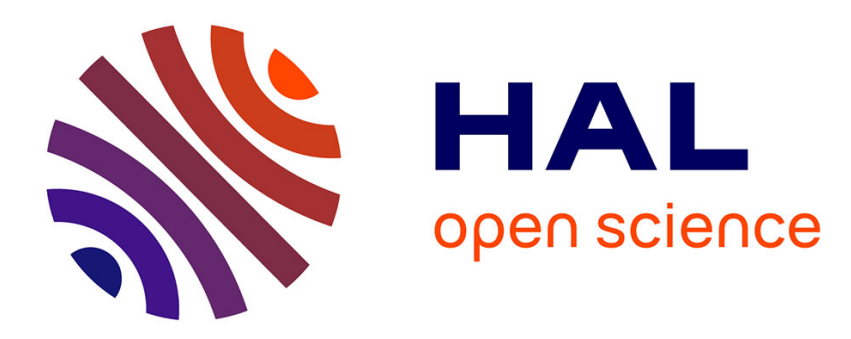

\title{
Divinités égyptiennes à Chypre à l'époque archaïque
}

Sabine Fourrier

\section{To cite this version:}

Sabine Fourrier. Divinités égyptiennes à Chypre à l'époque archaïque. Egypt and Cyprus in Antiquity, Apr 2003, Nicosie, Chypre. pp.97-103. hal-01453034

\section{HAL Id: hal-01453034 https://hal.science/hal-01453034}

Submitted on 2 Feb 2017

HAL is a multi-disciplinary open access archive for the deposit and dissemination of scientific research documents, whether they are published or not. The documents may come from teaching and research institutions in France or abroad, or from public or private research centers.
L'archive ouverte pluridisciplinaire HAL, est destinée au dépôt et à la diffusion de documents scientifiques de niveau recherche, publiés ou non, émanant des établissements d'enseignement et de recherche français ou étrangers, des laboratoires publics ou privés. 
This pdf of your paper in Egypt and Cyprus in Antiquity belongs to the publishers Oxbow Books and it is their copyright.

As author you are licenced to make up to 50 offprints from it, but beyond that you may not publish it on the World Wide Web until three years from publication (September 2012), unless the site is a limited access intranet (password protected). If you have queries about this please contact the editorial department at Oxbow Books (editorial@oxbowbooks.com). 



\title{
An offprint from
}

\section{Proceedings of the International Conference \\ EGYPT AND CYPRUS IN ANTIQUITY \\ Nicosia, 3-6 April 2003}

\author{
Edited by \\ D. Michaelides, V. Kassianidou and R. S. Merrillees
}





\section{Contents}

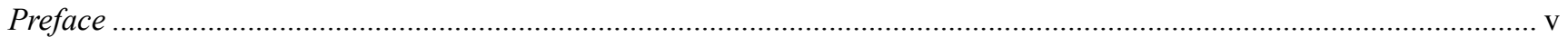

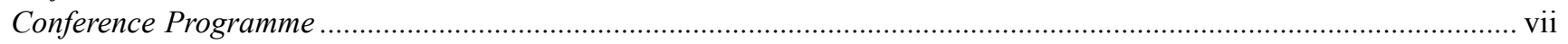

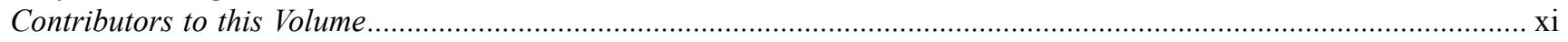

PART I. BRONZE AGE

edited by R. S. Merrillees and V. Kassianidou

1. Alas(h)i(y)a (Irs) and Asiya (Isy) in Ancient Egyptian Sources

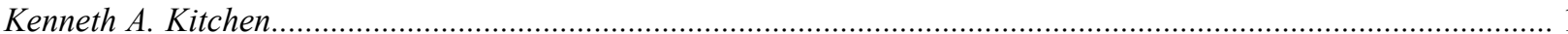

2. Peuples, états et cités. Enquête sur la cartographie géopolitique égyptienne

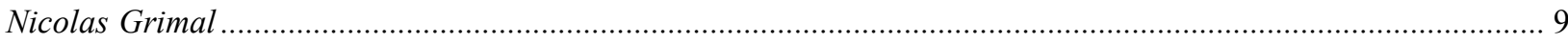

3. The Stone Vases of the Philia Culture from Vasilia: Cypriote, Egyptian or Other?

Robert S. Merrillees

4. A Contribution from Tell el-Dab ${ }^{c}$ a/ ${ }^{2}$ Ezbet Helmi to the Cyprus Connection at the Turn of Middle Bronze Age

to Late Bronze Age

Irmgard Hein.

5. Embracing the New: the Perception of Cypriot Pottery in Egypt

Linda Hulin

6. "May he send me silver in very great quantities" EA 35

Vasiliki Kassianidou

PART II. IRON AGE

edited by V. Kassianidou

7. The Relations between Egypt and Cyprus from Neo-Assyrian to Achaemenid Rule (7th-6th Cent. B.C.)

Andreas Mehl.

8. The Relations of Egyptian Iconography and Symbolism with the Royal Ideology of Cypriot City Kingdoms

Pavlos Flourentzos.

9. L'Égypte, Chypre et la route de Péluse à Gaza: approche micro-régionale des politiques stratégiques des derniers souverains égyptiens et achéménides (610-332 av. J.-C.)

Claire Balandier.

10. Divinités égyptiennes à Chypre à l'époque archaïque

Sabine Fourrier

11. From Siwa to Cyprus: The Assimilation of Zeus Ammon in the Cypriote Pantheon

Derek B. Counts 
12. Le Baal d'Amathonte et le Bès égyptien

Isabelle Tassignon

13. Representations of Cypriote Scribes and Carriers of Written Documents: a Link with Egypt?

Frieda Vandenabeele

PART III. CLASSICAL AND LATER PERIODS

edited by $D$. Michaelides

14. Un Apollon Chypriote aux Boucles « Libyques »

Antoine Hermary

15. Fusion and Diffusion. Isiac Cults in Ptolemaic and Roman Cyprus

Aristodemos Anastasiades

16. Ptolemaic Art and the Legitimation of Power

Emilia Dina Vassiliou

17. Les relations culturelles entre l'Égypte et Chypre: la céramique d'Alexandrie et de Paphos

Pascale Ballet

18. Modèles alexandrins ? Apport des fouilles et prospections récentes dans la nécropole occidentale d'Alexandrie à la connaissance de l'architecture funéraire hellénistique et impériale

Marie-Dominique Nenna.

19. Twilight of the Ptolemies: Egyptian Presence on Late Hellenistic Yeronisos

Joan Breton Connelly

20. Sailors and Artisans: the Egyptian Connections of Ceramic Finds from Yeronisos

Jolanta Młynarczyk

21. Alexandrian Influences on the Architecture and Decoration of the Hellenistic Tombs of Cyprus

Anne-Marie Guimier-Sorbets and Demetrios Michaelides

22. Alexandrian Elements in Cypriot Burial Customs of the Hellenistic and Roman Periods

†Danielle A. Parks

23. La contribution des conteneurs commerciaux à l'histoire des échanges entre Chypre et l'Égypte de l'èpoque archaïque à l'èpoque romaine tardive

Antigone Marangou et Sylvie Marchand

24. The Nile Flows Underground to Cyprus: the Painted Water-Cistern at Salamis Reconsidered

Helen Whitehouse 


\title{
10. Divinités égyptiennes à Chypre à l'époque archaïque
}

\author{
Sabine Fourrier
}

Les artisans chypriotes multiplient, à l'époque archaïque, les emprunts à l'art égyptien. Ces éléments égyptisants recouvrent une grande diversité de formes, depuis certains détails du vêtement ou de la coiffure jusqu'à la reprise d'images divines (Hermary 2001, 28), sur une grande variété de supports (céramique, petite et grande plastique), dans l'ensemble de l'île, aussi bien dans les royaumes côtiers, comme Amathonte (Petit 1995), qu'à l'intérieur (Buchholz 1993, pour Tamassos). Pourtant, les importations égyptiennes, à l'exception des petites faïences, sont très rares et elles ne sauraient rendre compte à elles seules de la profonde transformation du répertoire local.

Cette situation paradoxale a suscité des interprétations divergentes, portant à la fois sur le mode de transmission des modèles et sur la signification de ces emprunts. Selon certains, Chypre entre, à l'époque archaïque, en relation directe avec l'Égypte, que ce soit à l'occasion de la « conquête » de l'île par le pharaon Amasis (Gjerstad 1948), ou bien dès la fin du VII ${ }^{\mathrm{e}}$ siècle, lorsque le delta égyptien s'ouvre durablement aux marchands grecs et chypriotes (Hermary 2001). La déesse égyptienne Hathor aurait été identifiée avec la « grande déesse » de Chypre, notamment à Amathonte (Hölbl 1994, 272-273), cette assimilation achevant, à la faveur de contacts renoués, un rapprochement peut-être amorcé dès le Bronze Récent (Scandone-Matthiae 1994). D'autres soutiennent l'hypothèse d'un lien indirect, par l'intermédiaire de l'art phénicien : le développement d'un art «égyptisant» à Chypre correspondrait à une expansion phénicienne dans l'île à la fin du $\mathrm{VI}^{\mathrm{e}}$ et au $\mathrm{V}^{\mathrm{e}} \mathrm{s}$. av. J.-C. (Markoe 1990). Glanée dans les cartons des artisans phéniciens, l'image des divinités égyptiennes serait coupée de sa signification originelle (Michaelidou Nicolaou 1978).

Sans étendre l'enquête à l'ensemble du répertoire archaïque, je m'intéresserai à deux séries de production chypriote qui utilisent l'image de divinités égyptiennes et qui, parce qu'elles n'ont pas de parallèle dans le domaine syro-palestinien, offrent un cadre propice à l'étude.

\section{Les sculptures « chypro-ioniennes »}

Les sculptures « chypro-ioniennes » ou de « style mixte » sont des statuettes de calcaire, retrouvées en grand nombre dans des sanctuaires grecs, pour l'essentiel en Grèce de 1'Est, et à Naucratis (Fourrier 2001, avec la bibliographie antérieure). Elles sont rarement attestées sur la côte syropalestinienne, à Amrit ou à Sidon, et à Chypre, où les trouvailles paraissent concentrées dans le royaume de Salamine. Elles sont d'ailleurs toujours associées, dans leur contexte de découverte, avec des figurines de terre cuite de production salaminienne. Fabriquées entre le dernier tiers $\mathrm{du} \mathrm{VII}{ }^{\mathrm{e}}$ siècle et le milieu du siècle suivant environ, elles présentent un style éclectique, qui emprunte ses traits à l'art chypriote comme à différents styles de la Grèce de l'Est ou à l'art égyptien. Leur origine - lieu de création et artisans - comme leur destination - clientèle - sont très discutées. Sans entrer ici dans ce débat, je me contenterai de souligner les deux points suivants. La composante chypriote de ces œuvres, qu'il s'agisse du style, de la technique ou du matériau, est essentielle, et Chypre ne saurait être considérée seulement comme une source d'inspiration ou de vague influence. L'île joue certainement un rôle important dans la création de ce répertoire, plus vraisemblablement en raison des artisans qui sont responsables de cette production qu'en raison du contexte (selon Kourou et al. 2002, ces statuettes auraient été fabriquées à Chypre par un ou des ateliers grecs installés dans l'île). Par ailleurs, cette série est très cohérente: la plupart des types se retrouvent d'un lieu de découverte à l'autre, et les liens stylistiques entre les œuvres, qu'elles aient été exhumées à Rhodes, Samos, Milet ou Naucratis, sont nombreux, ce qui invite à ne pas multiplier le nombre des ateliers. La carte de diffusion de cette production, qui recoupe celle des cités les plus actives à Naucratis, conforte, en l'état actuel de la documentation, une localisation de l'atelier dans cette ville du delta égyptien où la présence de Chypriotes est attestée par les sources littéraires et archéologiques (Masson 1971, Davis 1979 et 1980). 
Si nombre de traits égyptisants du répertoire « chyproionien » trouvent des parallèles dans l'art syro-palestinien (représentations de sphinx ou de faucons, par exemple), certains y sont inconnus et suggèrent la reprise directe de modèles égyptiens. M. Yon l'a bien montré pour un groupe de petits lions découverts à Salamine (Yon 1974). Il est possible que la série des sculptures « chypro-ioniennes » ait joué le rôle de relais dans la transmission à Chypre de motifs égyptiens que l'artisanat phénicien ne connaît pas. Un groupe, découvert à Naucratis, représente un personnage féminin trônant, allaitant un enfant (Fig. 10.1). La façon tout à fait caractéristique de figurer l'enfant est citée, plus qu'elle n'est imitée, sur une série de figurines de terre cuite moulées, provenant de Lapithos (Yon et Caubet 1988, 6-8). La posture de l'enfant se retrouve sur des vases plastiques égyptiens de faïence représentant une femme accroupie tenant sur ses genoux un animal, plus rarement un enfant, et portant dans le dos un enfant maintenu dans une sorte de

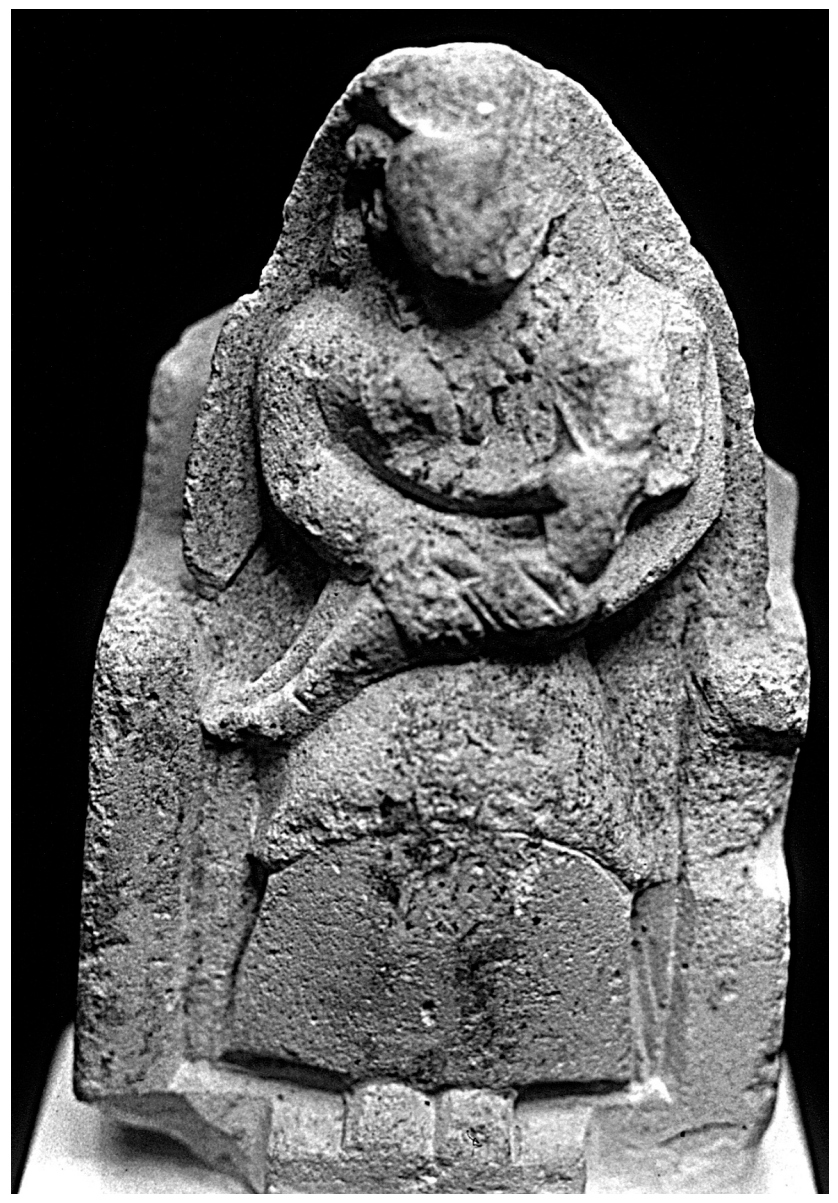

Fig. 10.1. Groupe de Naucratis, British Museum B 463 (d'après Pryce, F. N., 1928, Catalogue of Sculptures in the Department of Greek and Roman Antiquities of the British Museum I.1, Londres, 197, fig. 238). sac. Un vase anthropomorphe de ce type a été recueilli lors des fouilles du temple archaïque de Kition; d'autres exemplaires ont été retrouvés en Grèce de l'Est, notamment à Rhodes (Lagarce et Leclant 1976). Si l'état actuel de la documentation ne permet pas de décider entre l'une ou l'autre source d'inspiration pour les ateliers de Lapithos (modèle égyptien importé à Chypre ou élaboré en Égypte par des artisans chypriotes), il n'en reste pas moins que le mode de transmission du motif est assurément direct et ne doit rien à l'entremise phénicienne.

C'est également le cas d'un type divin nouveau, dont la série « chypro-ionienne » offre les premiers exemples et qui connaît à Chypre un succès certain, essentiellement à partir de la fin de l'archaïsme. Dans le schéma iconographique le plus ancien, illustré par les statuettes « chypro-ioniennes » et de rares exemples provenant de Salamine (Hermary 1992), le dieu, trônant, est criocéphale (Fig. 10.2). Les études que leur ont consacré des sémitisants ont exclu, de manière définitive, qu'il puisse s'agir de représentations de BaalHammon (Lipiński 1986, Xella 1991 ; contre Buchholz 1991). Mais l'appellation «Zeus-Ammon », généralement

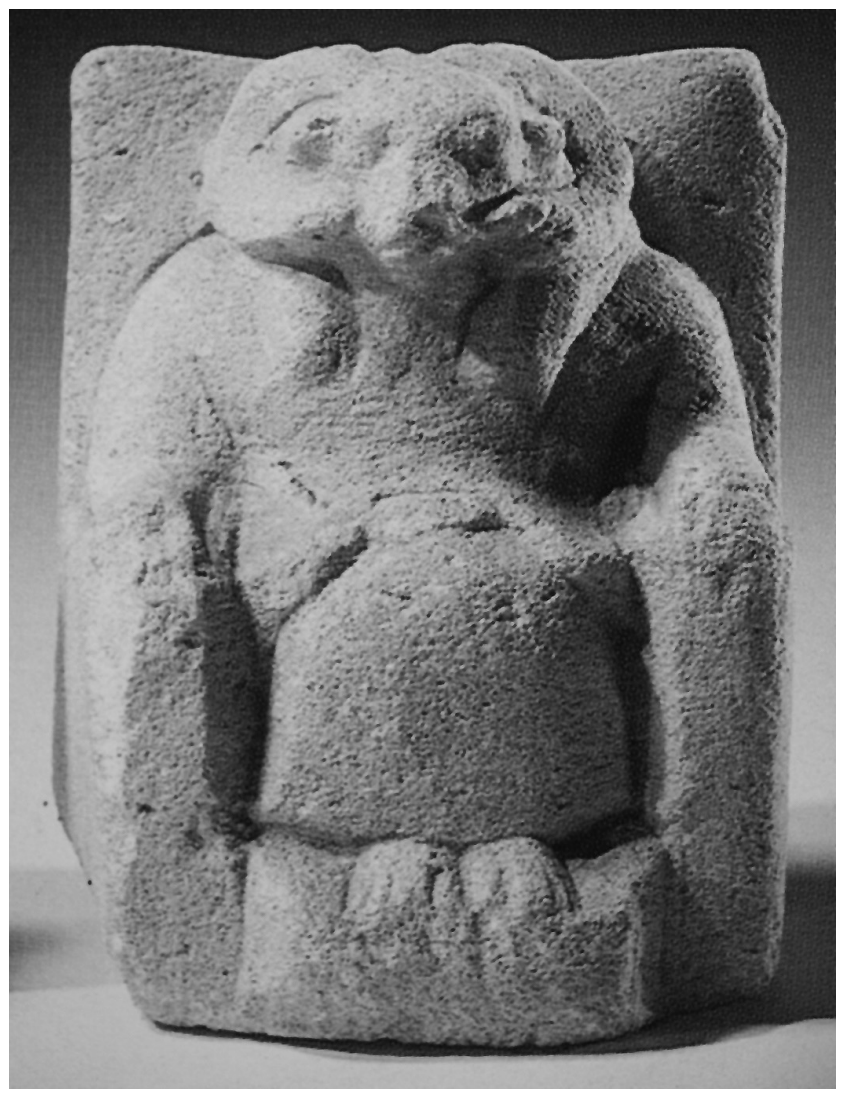

Fig. 10.2. «Zeus-Ammon » de Lindos, Copenhague Inv. $\mathrm{n}^{\circ} 10448$ (d'après Karageorghis, V. et al., 2001, Ancient Cypriote Art in Copenhagen, Nicosie, 82-83, no. 155). 
utilisée, est ambiguë. De fait, ces exemples chypriotes ne peuvent pas s'inscrire dans le cadre de diffusion du culte de Zeus-Ammon qui, depuis Cyrène, a essaimé, au cours de la période classique, dans le monde grec (Classen 1959). Zeus-Ammon qui est attesté, pour la première fois, à Cyrène et seulement à la fin du VI ${ }^{\mathrm{e}}$ s. av. J.-C., a toujours figure humaine (Chamoux 1953, 337). Les représentations « chyproioniennes », toujours criocéphales, sont assurément antérieures au milieu du $\mathrm{VI}^{\mathrm{e}}$ siècle, et elles prècèdent donc largement dans le temps le type dont elles seraient dérivées (Fourrier 2001, 41). Il est difficile de supposer, comme on l'a proposé (Lipiński 1986, 315), que les sculpteurs chypriotes aient directement repris le type iconographique du temple de Siwa, sur lequel on n'est guère informé et dont le rayonnement hors des frontières de l'Égypte ne paraît pas important à date aussi ancienne (Kuhlmann 1988, Letzner 2003). En revanche, les sculpteurs chypriotes, actifs à Naucratis à cette époque, ont pu prendre pour modèle l'image de la divinité tutélaire de la ville, Amon-Baded. Si la plupart des documents conservés sont d'époque ptolémaïque (Yoyotte 1982-1983), une stèle de donation semble montrer que le culte du dieu criocéphale était déjà florissant à Naucratis à l'époque saïte (Yoyotte 1991-1992, 643-644). Il est remarquable que les plus anciennes attestations du type à Chypre, en tous points semblables à celles que livre la série « chypro-ionienne », proviennent de Salamine, dont la principale divinité est Zeus (Yon 1981, Hermary 1992). C'est, en effet, en le nommant Zeus Thébaios que les Grecs s'adressent à l'époque archaïque, en Égypte, à l'Amon de Thèbes (Jeffery 1963, 355). Les artisans chypriotes ont su reprendre et interpréter une image adaptée à leur propos. Par la suite, cette image, qui se répand dans l'île sous une forme humanisée, subit sans doute l'influence du type cyrénéen, au moment où les relations entre la cour de Cyrène et celle d'Évelthon de Salamine sont étroites (Chamoux 1953, 144-159 ; Hermary 2001, 30) et les deux figures tendent à se confondre. Toutefois, s'il y a contamination iconographique, il n'y a pas d'implantation à Chypre du culte de Zeus-Ammon. L'image du dieu à tête puis seulement cornes de bélier, que les artisans chypriotes ont les premiers élaborée, est empruntée à bon escient, mais elle est celle d'une divinité locale.

\section{Les vases du « style d'Amathonte »}

À la fin de la période archaïque, les potiers amathousiens fabriquent une série très homogène de vases - des amphorisques et, pour quatre exemplaires connus seulement, des cruches (Hermary 1997, pl. LI = fig. 4 ; Karageorghis 1989, pl. 16, 1- 3 et pl. 17, 1-2 ; Karageorghis et des Gagniers 1974, 511, cat. $n^{\circ} 8$ ) -, qui se distinguent du reste de leur production par leur technique de décor et leur répertoire iconographique. Rarement réalisés dans les fabriques White Painted ou Bichrome Red, ces vases portent le plus souvent une décoration " pseudo-bichrome ", qui joue sur le contraste de deux teintes obtenues par cuisson réductrice (Karageorghis et des Gagniers 1974, 91-93). Sur les exemples les plus soignés, certaines zones décorées sont détaillées au moyen d'incisions qui imitent sommairement, dans leur effet, la manière grecque de la figure noire (Fig. 10.5). À la différence du reste de la production locale, le décor, souvent organisé en métopes sur la panse, est fréquemment figuré. Cette série, qui n'est guère exportée hors des frontières du royaume d'Amathonte, devait avoir une signification cultuelle locale. Seuls deux exemples d'origine douteuse proviendraient l'un de Phocée ou de Myrina (British Museum C $852=$ Karageorghis et des Gagniers 1974, p. 505, cat. $n^{\circ} 2$ ), l'autre d'Idalion (Musée National de Copenhague $\mathrm{n}^{\circ} 1059=$ Karageorghis et des Gagniers 1974, p. 509, cat. $n^{\circ}$ 6). La plupart des exemplaires ont été mis au jour à Amathonte (Hermary 1997), surtout dans les nécropoles de la ville, mais aussi dans le sanctuaire d'Aphrodite sur l'acropole et dans le palais (qui est le lieu d'origine probable du dépôt de la terrasse ouest et assuré de celui découvert contre le rempart nord de la ville basse). Quelques vases isolés ont été retrouvés dans des tombes à Limassol (Bulletin de Correspondance Hellénique, 101, 1977, 724, fig. 39 ; ibid., 106, 1982, 704, fig. 57 ; ibid., 111, 1987, 724, fig. 204) et à Kalavassos-Kafkallies (Bulletin de Correspondance Hellénique, 108, 1984, 925, fig. 111-112), deux sites qui appartenaient alors certainement au territoire d'Amathonte.

Le répertoire iconographique du « style d'Amathonte » est varié, mais relativement redondant, les motifs pouvant être employés de manière isolée ou en assemblage. Outre de quelques décors géométriques (croisillons et damiers), dont certains sont combinés avec des fleurs de lotus stylisées (British Museum C $850=C V A$ Royaume-Uni 2, pl. 12, 1, par exemple), les panses des vases s'ornent de motifs végétaux, dont des représentations de lotus, papyrus (Fig. 10.3) et palmiers ; d'animaux, poissons, coqs et chèvres; d'une composition d'éléments végétaux commnunément appelée « arbre sacré »; d'objets, comme des thymiateria ou, pour un exemple, d'un aryballe ; de sphinx et de têtes hathoriques (Figs 10.4 et 10.5). Quelques amphorisques portent des représentations plus ambitieuses, véritables scènes, souvent fragmentaires, mais dont le sens cultuel n'est pas douteux (Hermary 1986, 168-172 et 1997, 158). On a insisté, à juste titre, sur l'importance des emprunts, tant formels que thématiques, aux productions contemporaines de la Grèce de l'Est (Karageorghis 1989 et 1990). La frise végétale qui orne le bas de l'amphorisque C 853 du British Museum (Karageorghis et des Gagniers 1974 , p. 507 , cat. $n^{\circ} 4$ ) ou le coq que porte la panse de l'amphorisque d'Amathonte T. 470/43 (Karageorghis 1989, pl. 17, 3) y renvoient certainement. D'autres motifs sont inspirés par le répertoire phénicien, ivoires et surtout coupes de métal précieux dont nombre d'exemples ont été découverts 


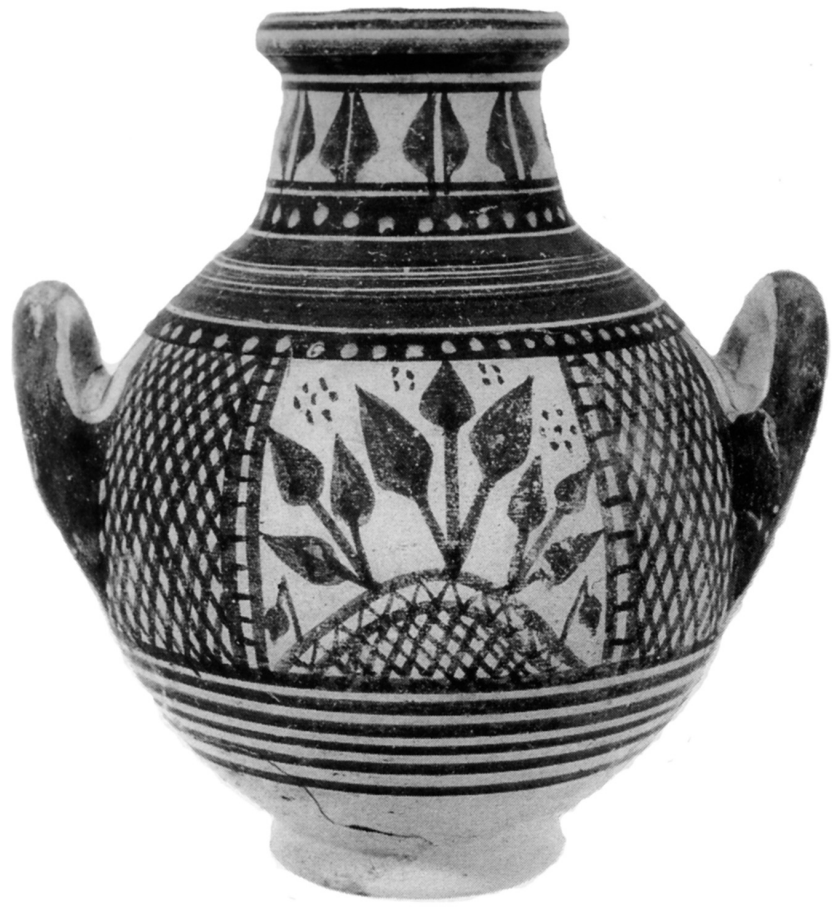

Fig. 10.3. Amphorisque d'Amathonte T. 204/27, musée de Limassol (d'après Hermary 1997, pl. LII e).

à Chypre : c'est, par exemple, le cas des représentations d'un « arbre sacré » qui combine de manière originale des éléments végétaux d'origine égyptienne (Shefton 1989, 98). Une même fidélité aux modèles, malgré l'écart qu'entraînent la transposition sur un support différent et la qualité, très variable, du dessin, est sensible dans la reprise de motifs égyptiens. Les frises denses qui ornent le col et l'épaule de beaucoup d'amphorisques ont été rapprochées de la « collerette » caractéristique des gourdes du Nouvel An égyptiennes (Lagarce et Leclant 1976, 237). Si le bosquet de papyrus qui décore la panse de l'amphorisque d'Amathonte T. 204/27 (Fig. 10.3) n'évoque que de façon lointaine les prototypes égyptiens, les deux tiges cassées de part et d'autre en sont une citation exacte (Hermary 1997, 160). L'emprunt le plus évident est celui de la tête hathorique, dont le traitement est là encore très variable, schématique ou soigneusement détaillé. La référence aux modèles égyptiens apparaît cependant clairement dans un certain nombre de détails formels. Ainsi, les têtes hathoriques sont souvent flanquées de motifs végétaux, qui ne l'encadrent pas à proprement parler, mais qui semblent surgir du bas du masque (Fig. 10.4). Il en est de même sur certaines représentations égyptiennes, par exemple sur des plaquettes de faïence de Serabit el-Khadim, sur lesquelles des tiges surmontées de boutons de lotus sont attachées à l'espèce de pilier trapézoïdal qui supporte la tête (Pinch 1993, pl. 31).

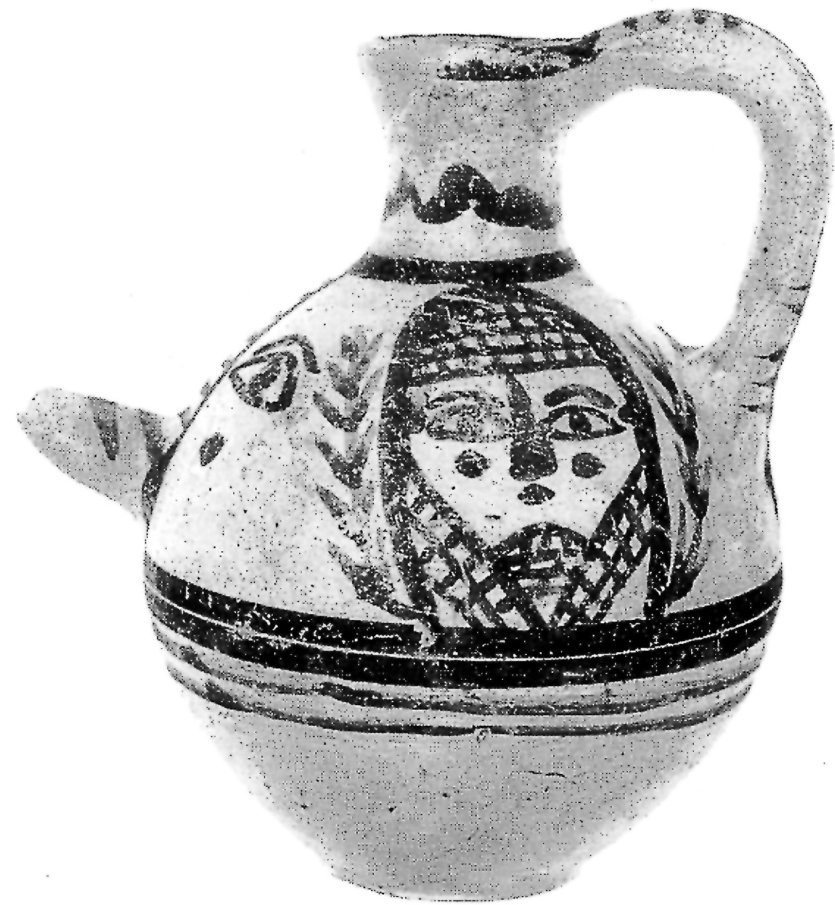

Fig. 10.4. Cruche d'Amathonte T. 194/18, musée de Limassol (d'après Hermary 1997, pl. LI b).

Ce « pilier » est d'ailleurs représenté sur certains exemples (Fig. 10.5) et il ne peut pas être confondu avec le couronnement papyriforme d'une stèle, illustré par ailleurs (notamment sur le tesson du Louvre AM 393 D = Karageorghis et des Gagniers 1974, p. 510, cat. $\mathrm{n}^{\circ} 7$ ). Les têtes hathoriques que portent les vases du «style d'Amathonte » ne sont donc pas toutes des représentations, plus ou moins abrégées, des stèles hathoriques que produisent, à la même époque, les sculpteurs chypriotes. Cet appendice trapézoïdal, parure, barbe ou colonnette (Pinch 1993, 135-159), qui signe les prototypes égyptiens, est connu dans l'île au Bronze Récent par une série de plaques en os décorées de masques hathoriques, mises au jour à Enkomi (Courtois et al. 1986, 130) et Toumba tou Skourou (Vermeule et Wolsky 1977, pl. XVIII). Ces quelques citations de motifs trahissent le recours direct à des modèles égyptiens.

Malgré l'éclectisme des sources, la relative limitation du répertoire iconographique suggère que ce dernier répond à un programme cohérent. Or, nombre de thèmes communs unissent les vases du « style d'Amathonte » avec une série de coupes et vases de faïence produits en Égypte au Nouvel Empire et importés dans l'île au Bronze Récent (Caubet et Lagarce 1972). On retrouve, en effet, les têtes hathoriques, la végétation de marais, lotus et papyrus, ou encore les représentations de poissons (von Bissing 1902, Krönig 1934, Dambach et Wallert 1966). Tous ces motifs renvoient au 


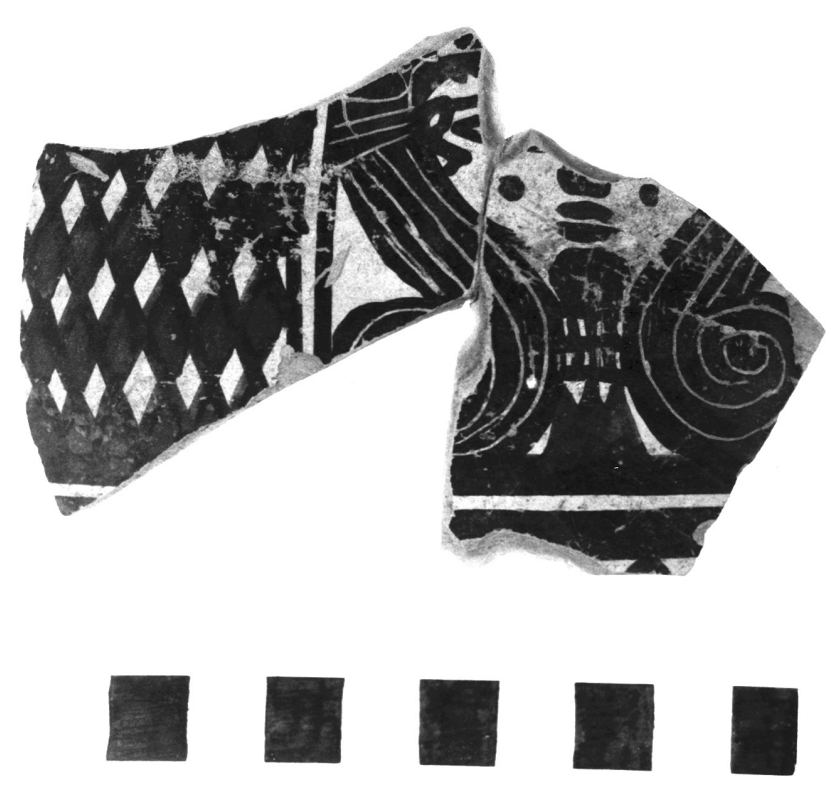

Fig. 10.5. Fragment découvert dans le dépôt du rempart nord d'Amathonte (cliché EFA, Ph. Collet).

thème de la fertilité, de la renaissance à laquelle préside Hathor, et ils sont bien adaptés à la personnalité de la " grande déesse " amathousienne, plus tard nommée Aphrodite. Cette renaissance est liée, dans le contexte égyptien, aux prérogatives funéraires de la déesse Hathor, Maîtresse de l'Occident (Pinch 1993, en particulier 120130). C'est ainsi que l'on voit apparaître, sur certaines coupes égyptiennes, des gazelles ou autres animaux du désert au milieu des papyrus et des poissons (Krönig 1934, 162, fig. 29 et 164, fig. 30). Plutôt que comme une représentation naturaliste - la gazelle viendrait boire à la rivière -, il faut interpréter ces scènes comme une association symbolique - mort et renaissance-, d'ailleurs connue par d'autres petits monuments hathoriques (Desroches-Noblecourt 1953). Or, les connotations funéraires de la déesse amathousienne sont documentées par Plutarque (Thésée, 20), qui rapporte l'existence d'un culte d'Ariane-Aphrodite, dont la tombe a peut-être été mise au jour, et par la découverte d'une grotte cultuelle dans le sanctuaire archaïque (Hermary 1994). Les emprunts à d'autres répertoires, grecs ou phéniciens, renvoient à la même symbolique : sphinx affrontés de part et d'autre d'un « arbre de vie », représentations de coqs de grande taille, proches de celles qui ornent certains sarcophages de Clazomènes (Cook 1981, p. 114 et n. 37, qui refuse toutefois toute interprétation symbolique). D'autres motifs, comme le palmier portant des régimes de dattes (par exemple sur l'amphorisque d'Amathonte T. 204/30 = Bulletin de Correspondance Hellénique, 105, 1981, 1010, fig. 85) ou les grenades (vase de provenance inconnue, UCLMA 8/3262 = Karageorghis et Amyx 1974,
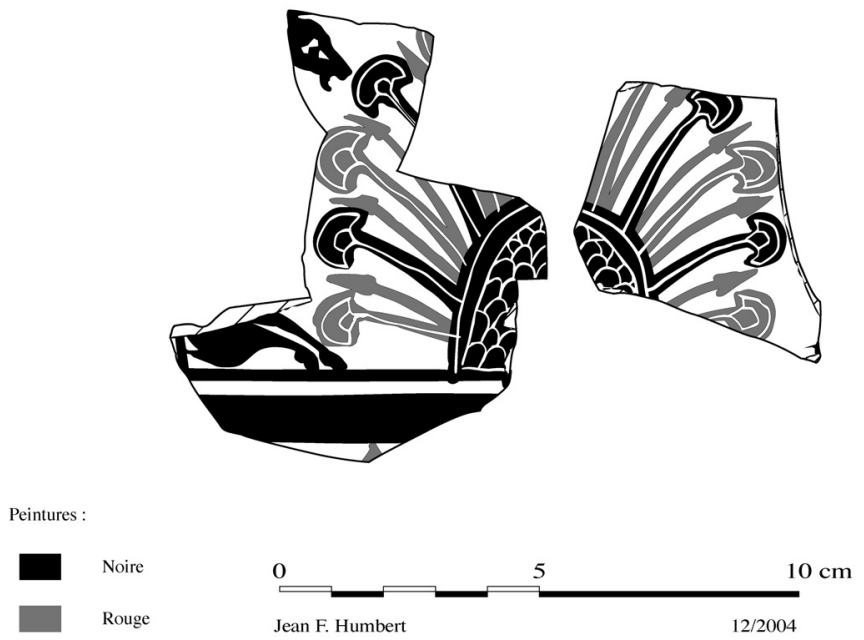

Fig. 10.6. Amphorisque fragmentaire découvert dans le dépôt du rempart nord d'Amathonte, inv. d/a.bichr.14 (dessin EFA, J. F. Humbert).

fig. 34), sont sans doute issus du répertoire local et ils se prêtent à une même lecture.

La déesse, souvent représentée par la tête hathorique, peut être évoquée sous une forme végétale, notamment sous celle d'un « arbre sacré ». Il est intéressant, à cet égard, de suivre les variations de ce motif dans la série des vases amathousiens. Au centre de la panse sur l'amphorisque de Princeton (Karageorghis 1990) ou au revers de celui du British Museum, où il remplace le masque hathorique (British Museum C $853=$ Karageorghis et des Gagniers 1974, 507, cat. $n^{\circ} 4$ ), cet « arbre sacré » reste proche des prototypes syro-palestiniens. Sur d'autres vases, il peut être abrégé en un triangle surmonté d'une fleur de lotus (British Museum C 963 = CVA Royaume-Uni 2, pl. 12, 10 et, de manière plus fruste, Cyprus Museum B $22=$ Karageorghis et des Gagniers 1974, p. 513, cat. n 10), d'une palmette (Newcastle-upon-Tyne, University Museum inv. $119=$ Karageorghis 1989, pl. 16, 1-3) ou de grenades (UCLMA 8/3262 = Karageorghis et Amyx 1974, fig. 34) ou bien en une sorte de « monticule » grillagé supportant des papyrus en bouton (Amathonte T. 204/27, Fig. 10.3). Ce « monticule » aux papyrus est un élément des «arbres sacrés » plus complexes que portent d'autres vases du «style d'Amathonte », tel l'amphorisque du British Museum déjà mentionné (British Museum C $853=$ Karageorghis et des Gagniers 1974, 507, cat. $\left.n^{\circ} 4\right)$. Il est également connu par des représentations en pierre, comme les chapiteaux dits proto-éoliques (Shiloh 1979, pl. 17, 1), où il évoque sans doute un plan d'eau. Pourtant, il prend en l'occurrence des proportions beaucoup plus importantes, et il s'orne d'un 
décor de quadrillage ou d'écailles qui, loin d'évoquer l'eau, paraît bien plutôt faire référence à une colline ou une montagne. De fait, la convention qui consiste à figurer une montagne sous la forme d'un monticule, arrondi ou triangulaire, et portant un décor de quadrillage est ancienne dans l'art proche-oriental : elle apparaît déjà sur un sceau akkadien provenant de Chypre et aujourd'hui dans la collection de George et Néféli Giabra Piéridès (Merrillees 2002,269 , cat. $\mathrm{n}^{\circ} 356$ ) ; on la retrouve au bandeau de la coupe de Kourion où elle sert à camper le décor montagneux de la scène de chasse (Markoe 1985, Cy7). Il est donc possible qu'en détournant ce motif, les peintres amathousiens aient fait référence à un autre thème hathorique égyptien, qui combine, dans un raccourci iconographique, la montagne, domaine funéraire, et le marécage, figuré par le bosquet de papyrus, lieu de la renaissance (Keimer 1956 ; Pinch 1993, 160-183).

Si les modèles sont donc divers, les peintres amathousiens ont toutefois su les utiliser et les interpréter avec cohérence. Un fragment, récemment découvert contre le rempart nord d'Amathonte, illustre parfaitement cette rencontre d'influences (Fig. 10.6) : la composition, qui figure deux chèvres affrontées de part et d'autre d'un "monticule », reprend le schéma proche-oriental des animaux de part et d'autre d'un " arbre sacré »; les chèvres sont empruntées au répertoire des peintres de la Grèce de l'Est, tandis que l'élément central renvoie à l'Égypte. La symbolique hathorique n'est pas simple répertoire décoratif, elle est employée pour son contenu. La récurrence de ces thèmes sur les vases du " style d'Amathonte " est peut-être liée à leur destination, fioles contenant un liquide aux vertus précieuses, à l'image des gourdes du Nouvel An égyptiennes (Lagarce et Leclant 1976, 237). Elle convient, en tout cas, à la personnalité de la " grande déesse » amathousienne qui, si elle peut prendre le visage d'Hathor, ne se confond pas avec elle.

La transformation du répertoire iconographique chypriote à l'époque archaïque est donc bien liée, du moins pour les exemples étudiés, à la reprise de contacts directs entre l'île et la vallée du Nil. L'impulsion décisive est sans doute davantage due à l'installation de Grecs et de Chypriotes dans le delta égyptien, à Naucratis, qu'à une éventuelle domination égyptienne sur l'île à l'époque d'Amasis. Le choix des motifs et leur interprétation locale témoignent d'une bonne connaissance du langage symbolique égyptien. Pour reprendre la formule employée par A. Caubet et E. Lagarce à propos des thèmes hathoriques, "Chypre est perméable non seulement au vocabulaire formel égyptien, mais également à son contenu symbolique ; celui-ci, peutêtre mieux compris qu'on ne l'a dit, a dû être adapté à des croyances et à des cultes locaux qui ont pu conserver dans certains rites, usages ou traditions, l'emploi d'objets, soit égyptiens, soit inspirés par l'Égypte. » (Caubet et Lagarce 1972, 127).
De fait, il n'y a pas, à l'époque archaïque, de culte égyptien à Chypre. L'image de divinités égyptiennes est assimilée, non pas les divinités elles-mêmes. Cette image n'est d'ailleurs jamais exclusive. Au moment où les peintres amathousiens reprennent le visage d'Hathor, les coroplathes du royaume préfèrent reproduire une silhouette de femme nue, se pressant les seins, aux lointains antécédents orientaux (Hermary 2000, 82-91). De même, cette image n'est pas pérenne : le Zeus de Salamine, représenté sous la forme d'un «Zeus-Ammon » à l'époque archaïque, est entièrement hellénisé au cours de l'époque classique, du moins dans le culte officiel, au moment où Isocrate loue le philhellénisme du roi Évagoras (Yon 1981).

\section{Bibliographie}

von Bissing, Fr. W., 1902, Catalogue général des Antiquités égyptiennes du musée du Caire, Fayencegefässe. Vienne.

Buchholz, H.-G., 1991, Der Gott Hammon und Zeus Ammon auf Zypern, Mitteilungen des Deutschen Archäologischen Institus, Athenische Abteilung, 106, 85-128.

Buchholz, H.-G., 1993, Ägyptisierendes aus Tamassos, Report of the Department of Antiquities, Cyprus, 195-206.

Caubet, A. et Lagarce, E., 1972, Vases en faïence de Chypre, Report of the Department of Antiquities, Cyprus, 113-128.

Chamoux, Fr., 1953, Cyrène sous la monarchie des Battiades, BEFAR, 170. Paris.

Classen, C. J., 1959, The Libyan god Ammon in Greece before 331 B.C., Historia, 8, 349-355.

Cook, R. M., 1981, Clazomenian Sarcophagi, Kerameus, 3. Mayence.

Courtois, J.-Cl., Lagarce, J., et Lagarce, E., 1986, Enkomi et le Bronze Récent à Chypre. Nicosie.

Dambach, M. et Wallert, I., 1966, Das Tilapia-Motiv in der altägyptischen Kunst, Chronique d'Égypte, 41, 273-294.

Davis, W., 1979, Ancient Naukratis and the Cypriotes in Egypt, Göttinger Miszellen, 35, 13-23.

Davis, W., 1980, The Cypriotes at Naukratis, Göttinger Miszellen, 41, 7-19.

Desroches-Noblecourt, Chr., 1953, Un « lac de turquoise ». Godets à onguents et destinées d'outre-tombe dans l'Égypte ancienne, Monuments Piot, 47, 1-34. Paris.

Fourrier, S., 2001, Naucratis, Chypre et la Grèce de l'Est: le commerce des sculptures « chypro-ioniennes », in U. Höckmann et D. Kreikenbom (eds), Naukratis. Die Beziehungen zu Ostgriechenland, Ägypten und Zypern in archaischer Zeit, 39-54. Möhnesee.

Gjerstad, E., 1948, The Swedish Cyprus Expedition, vol. 4, part 2, The Cypro-Geometric, Cypro-Archaic and Cypro-Classical Periods. Stockholm.

Hermary, A., 1986, Divinités chypriotes II, Report of the Department of Antiquities, Cyprus, 164-172.

Hermary, A., 1992, Représentations de Zeus-Ammon à Chypre: à propos d'un article récent, Cahier du Centre d'Études Chypriotes, 18, 15-20.

Hermary, A., 1994, La tombe du sanctuaire d'Aphrodite à 
Amathonte, Report of the Department of Antiquities, Cyprus, 197-201.

Hermary, A., 1997, Le « style d'Amathonte », in V. Karageorghis, R. Laffineur et Fr. Vandenabeele (eds), Four Thousand Years of Images on Cypriote Pottery, 157-161. Bruxelles-LiègeNicosie.

Hermary, A., 2000, Amathonte V, Les figurines de terre cuite archaïques et classiques, les sculptures en pierre, Études Chypriotes XV. Paris.

Hermary, A., 2001, Naucratis et la sculpture égyptisante à Chypre, in U. Höckmann et D. Kreikenbom (eds), Naukratis. Die Beziehungen zu Ostgriechenland, Ägypten und Zypern in archaischer Zeit, 27-37. Möhnesee.

Hölbl, G., 1994, Vorhellenistische Isisfigürchen des ägäischen Raumes, insbesondere von der Insel Rhodos, in C. Berger, G. Clerc et N. Grimal (eds), Hommages à Jean Leclant, 271283. Le Caire.

Jeffery, L. H., 1963, The Local Scripts of Archaic Greece. Oxford.

Karageorghis, V., 1989, Some remarks on the «Amathus Style» in Cypriote vase-painting, in H.-U. Cain, H. Gabelmann et D. Salzmann (eds), Festschrift für Nikolaus Himmelmann, 83-86. Mayence.

Karageorghis, V., 1990, The Princeton amphoriskos of the Amathus style, Report of the Department of Antiquities, Cyprus, 121125.

Karageorghis, V. et Amyx, D. A., Corpus of Cypriote Antiquities 5, Cypriote Antiquities in San Francisco Bay Area Collections, SIMA XX: 5. Göteborg.

Karageorghis, V. et des Gagniers, J., 1974, La céramique chypriote de style figuré. Rome.

Keimer, L., 1956, La vache et le cobra dans les marécages de papyrus de Thèbes, Bulletin de l'Institut d'Égypte, 37, 215257.

Kourou, N., Karageorghis, V., Maniatis, Y., Polikreti, K., Bassiakos, Y. et C. Xenophontos, 2002, Limestone Statuettes of Cypriote Type Found in the Aegean. Provenance Studies. Nicosie.

Krönig, W., 1934, Ägyptische Fayence-Schalen des Neuen Reiches, Mitteilungen des Deutschen Archaölogischen Instituts, Abteilung Kairo, 5, 144-166.

Kuhlmann, Kl. P., 1988, Das Ammoneion. Archäologie, Geschichte und Kultpraxis des Orakels von Siwa. Mayence.

Lagarce, E. et Leclant, J., Vase plastique en faïence KIT. 1747: une fiole pour eau de jouvence, in G. Clerc et al, Fouilles de Kition II, Objets égyptiens et égyptisants, 183-290. Nicosie.

Letzner, W., 2003, Kultstätten im Dienste des Ammon-Re, Antike Welt, 34, 49-58.
Lipiński, E., 1986, Zeus-Ammon et Baal-Hammon, in C. Bonnet, E. Lipiński et P. Marchetti (eds), Studia Phoenicia IV, Religio Phoenicia, 307-332. Namur.

Markoe, Gl., 1985, Phoenician Bronze and Silver Bowls from Cyprus and the Mediterranean. Berkeley.

Markoe, Gl., 1990, Egyptianizing male votive statuary from Cyprus: a re-examination, Levant, 22, 111-122.

Masson, O., 1971, Les Chypriotes en Égypte, Bulletin de la Société Française d'Égyptologie, 60, 28-45.

Merrillees, P. H., 2002, Cylinder seals, in V. Karageorghis, Ancient Art from Cyprus in the Collection of George and Nefeli Giabra Pierides, 265-285. Athènes.

Michaelidou-Nicolaou, I., 1978, The cult of oriental divinities in Cyprus, in M. B. de Boeret et T. A. Edridge (eds), Hommages à M.J. Vermaseren, II, Leyde, Études Préliminaires aux Religions Orientales 68, 791-800. Leyde.

Petit, Th., 1995, Objets égyptisants et idéologie royale à Amathonte, Transeuphratène, 9, 131-147.

Pinch, G., 1993, Votive Offerings to Hathor. Oxford.

Scandone-Matthiae, G., 1994, L'Oro et la Dorata. Un' ipotesi su un epiteto di Aphrodite e di Hathor, in C. Berger, G. Clerc, et N. Grimal (eds), Hommages à Jean Leclant, 435-440. Le Caire.

Shefton, B. B., 1989, The paradise flower, a 'Court Style' Phoenician ornament: its history in Cyprus and the Central and Western Mediterranean, in V. Tatton-Brown (ed.), Cyprus and the East Mediterranean in the Iron Age, 97-111. Londres.

Shiloh, Y., 1979, The Proto-Aeolic Capital and Israelite Ashlar Masonry, Qedem, 11. Jérusalem.

Vermeule, E. T. et Wolsky, Fl. Z., 1977, The bone and ivory of Toumba tou Skourou, Report of the Department of Antiquities, Cyprus, 80-96.

Xella, P., 1991, Baal Hammon. Recherches sur l'identité d'un dieu phénico-punique. Rome.

Yon, M., 1974, Les lions archaïques, in Salamine de Chypre IV, Anthologie Salaminienne, 5-12. Paris.

Yon, M., 1981, Du taureau à l'aigle, in L. Kahil et Chr. Augé (dir.), Mythologie gréco-romaine, mythologies périphériques, 89-93. Paris.

Yon, M. et Caubet, A., 1988, Un culte populaire de la grande déesse à Lapithos, Report of the Department of Antiquities, Cyprus, 1-16.

Yoyotte, J., 1982-1983, L'Amon de Naucratis, Revue d'Égyptologie, 34, 129-136.

Yoyotte, J., 1991-1992, Égyptologie, Annuaire du Collège de France, 639-644. 